\title{
THEORY OF MAGNETOTUNNELLING THROUGH DONORS IN THE QUANTUM WELL OF DOUBLE-BARRIER RESONANT-TUNNELLING STRUCTURES
}

\author{
T.M. Fromhold, F.W. Sheard and L. Eaves \\ Department of Physics, University of Nottingham, Nottingham, NG7 2RD, U.K.
}

The tunnel current from a two-dimensional electron gas (2DEG) into donor impurities in the quantum well of a double-barrier heterostructure is studied using the Bardeen Transfer-Hamiltonian formalism. Resonant tunnelling occurs when the donor level lies within the energy range of occupied 2DEG states. It is shown that in the presence of a magnetic field $\boldsymbol{B}$ normal to the current flow, the magnetocurrent $J(B)$ is determined by the Fourier probability density of the donor wave function corresponding to the momentum $p_{0}$ transferred to the tunnelling electrons by the Lorentz force. Then experimental $J(B)$ curves, in principle, directly measure the donor wave function. The magnetocurrent is quenched when $p_{0}$ greatly exceeds the width of the distribution of momentum Fourier components of the donor wave function.

PACS numbers: 73.40.Gk, 71.55.Eq, 85.30.Tv

The precision growth of semiconductor heterostructures has permitted systematic studies of a wide range of quantum phenomena. In particular, quantum interference and high-energy electron dynamics have been extensively investigated using double-barrier resonant-tunnelling structures (DBRTS) in which the electrons are injected from a two-dimensional electron gas (2DEG) into the subband states of the quantum well. The injection energy is determined by the applied bias voltage. Using a transverse magnetic field (perpendicular to the current flow) to control the momentum of the injected carriers, the magnetotunnelling rates and lateral energy dispersion curves have been determined for electrons and holes [1-3].

Recent experiments on laterally-gated DBRTS and on GaAs/(AlGa)As DBRTS containing an $n$-type $\delta$-doped layer in the middle of the quantum well, have revealed resonances in the current-voltage curve below the threshold voltage for resonant tunnelling through the lowest subband minimum of the well $[4,5]$. These resonances are suppressed by a transverse magnetic field $\approx 10 \mathrm{~T}$ and originate from tunnelling through bound states of the donor impurities.

We assume that the donors in the quantum well are essentially non-interacting since in the experiments, the nearest-neighbour separation greatly exceeds the 
Bohr radius in GaAs [5]. We therefore use the Bardeen Transfer-Hamiltonian approach $[2,6]$ to calculate the voltage- and field-dependence $J(V, B)$ of the current through a single donor.

Figure 1 shows the conduction band profile of the $\delta$-doped structures. Using the vector potential in the Landau gauge $\boldsymbol{A}=\left(0, B_{x}, 0\right)$, the magnetic field only slightly perturbs the $2 \mathrm{D}$ electrons which retain free lateral motion [6]. Taking the

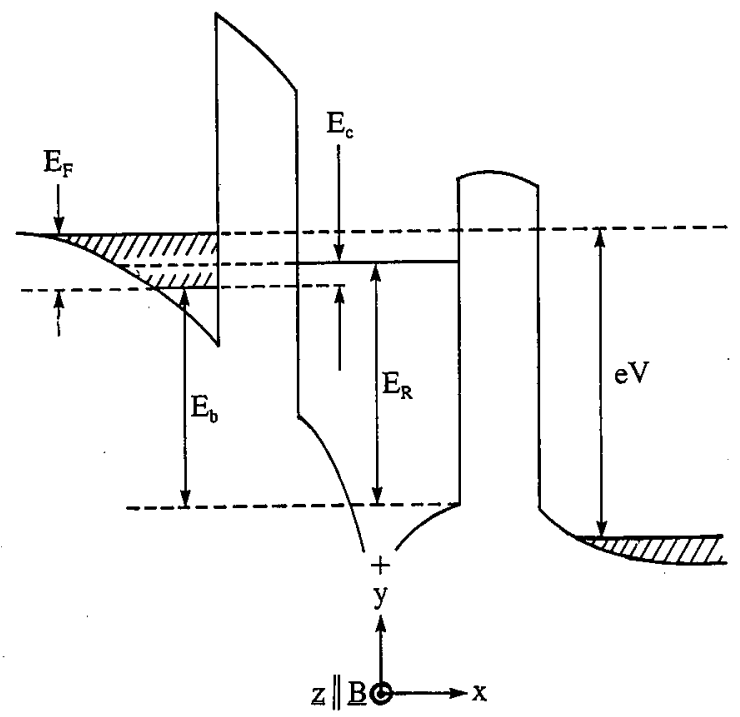

Fig. 1. Conduction band profile of the $\delta$-doped structures. The donor ground state energy $E_{R}$ is approximately $12 \mathrm{meV}$ below the lowest subband minimum of the quantum well [8].

origin of the coordinates $x=0$ at the donor site as in Fig. 1, the perturbed energy levels in the emitter are $[2,6]$

$$
E_{\mathrm{L}}\left(p_{y}, p_{z}\right)=E_{\mathrm{b}}+\frac{\left(p_{y}-p_{0}\right)^{2}}{2 m^{*}}+\frac{p_{z}^{2}}{2 m^{*}}
$$

where the bound-state energy $E_{\mathrm{b}}$ includes a small diamagnetic shift, $\boldsymbol{p}=\left(p_{y}, p_{z}\right)$ is the lateral canonical momentum, and $p_{0}=B e \Delta s$ is the kinetic momentum transferred classically as the electrons traverse the mean distance $\Delta s$ between the 2DEG and the donor impurity [7]. The origin of the parabolic dispersion relations is thus shifted to $\left(p_{0}, 0\right)[2,6,7]$. Energy conservation in the tunnelling process is satisfied by those states for which $E_{\mathrm{L}}\left(p_{y}, p_{z}\right)=E_{R}$, where $E_{R}$ is the energy of the donor electron as in Fig. 1. This gives $\left(p_{y}-p_{0}\right)^{2}+p_{z}^{2}=p_{c}^{2}$, where $E_{c}=p_{c}^{2} / 2 m^{*}=$ $E_{R}-E_{\mathrm{b}}$ is the lateral kinetic energy of the resonant electrons in the 2DEG. The momentum $p_{c}$ decreases with increasing voltage as the donor level sweeps down through the emitter states. The bardeen transition rate from the 2DEG into the donor ground state $\Psi(x, y, z)=\psi(x) \phi(y) \chi(z)$, assumed field-independent and of separable form [8], is

$$
W\left(p_{y}, p_{z}\right)=N\left|F\left(p_{y}\right)\right|^{2}\left|G\left(p_{z}\right)\right|^{2} \delta\left(E_{\mathrm{L}}-E_{R}\right)
$$


where $F\left(p_{y}\right), G\left(p_{z}\right)$ are the Fourier transforms of $\phi(y)$ and $\chi(z)$, respectively. The prefactor $N$ depends on the $x$-dependent decay of the 2DEG and donor wave functions in the barrier and is only weakly field-dependent when the barrier is narrow [2]. We assume that the tunnelling rates (2) are small compared with the rates of scattering processes in the well so that the occupancies of the donor states are almost zero. Integrating Eq. (2) over the energy-conserving 2DEG states and neglecting level broadening, gives the current density per donor

$$
J(V, B)=\frac{4 N e m^{*}}{h^{2}} \int_{p_{0}-p_{c}}^{p_{0}+p_{c}} \frac{\left|F\left(p_{y}\right)\right|^{2}\left|G\left(p_{z}\right)\right|^{2} \mathrm{~d} p_{y}}{\left|p_{z}\right|},
$$

where $p_{z}^{2} / 2 m^{*}=\left[p_{c}^{2}-\left(p_{y}-p_{0}\right)^{2}\right] / 2 m^{*}$ is defined by energy conservation. Current flows over the voltage range $V_{1} \leq V \leq V_{2}$ for which the donor level is aligned with occupied emitter states. Assuming Gaussian wave functions $\phi(y)=\exp \left(-y^{2} / \lambda^{2}\right)$ and $\chi(z)=\exp \left(-z^{2} / \lambda^{2}\right)$ [8], Eq.(3) predicts that when no magnetic field is applied

$$
J(V, 0)=J_{0} \exp \left(-\lambda^{2} p_{c}^{2} / 2 \hbar^{2}\right) \quad\left(V_{1} \leq V \leq V_{2}\right)
$$

where $J_{0}$ depends on device parameters. Thus $J(V, 0)$ rises almost exponentially with voltage as $p_{c}$ falls from $p_{\mathrm{F}}=\left(2 m^{*} E_{\mathrm{F}}\right)^{1 / 2}$ under bias $V_{1}$, down to zero when $V=V_{2}$ and the emitter and donor bound-state energies coincide. The ratio $J\left(V_{2}, 0\right) / J\left(V_{1}, 0\right)=\exp \left(\lambda^{2} p_{\mathrm{F}}^{2} / 2 \hbar^{2}\right)$ depends on the spatial extent of the lateral donor wave function.

Figure 2 shows the Fourier probability distribution $\left|F\left(p_{y}\right)\right|^{2}$ and the factor $\left|G\left(p_{z}\right)\right|^{2} /\left|p_{z}\right|$ which determine the integrand of Eq. (3). The horizontal line shows

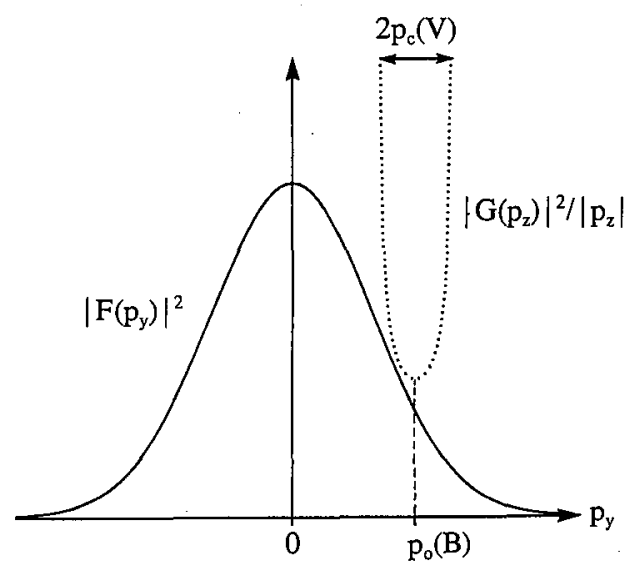

Fig. 2. Schematic Fourier probability density $\left|F\left(p_{y}\right)\right|^{2}$. Horizontal line shows the range of integration in Eq. (3). The bias controls the length of the line and the magnetic field determines the offset $p_{0}$. The broken curve shows $\left|G\left(p_{z}\right)\right|^{2} /\left|p_{z}\right|$. With increasing $B$ this curve is shifted along the $p_{y}$-axis but its shape is unchanged.

the range of integration. Increasing the magnetic field at fixed bias shifts this range to higher $p_{y}$ values for which $\left|F\left(p_{y}\right)\right|^{2}$ is smaller, thereby suppressing the current (3). Note that increasing $B$ shifts the origin $p_{0}$ of $\left|G\left(p_{z}\right)\right|^{2} /\left|p_{z}\right|$ but does not change 
its form over the range of integration. The current fall-off is therefore entirely due to the diminished Fourier components $\left|F\left(p_{y}\right)\right|^{2}$. The current is essentially quenched when the range of integration passes beyond the range of appreciable Fourier components.

When the emitter and donor bound-state energies coincide under bias $V_{2}$ only electrons with zero lateral momentum tunnel and the horizontal line in Fig. 2 shrinks to a point. Classically these electrons reach the centre of the well with a unique lateral kinetic momentum $\left(p_{0}, 0\right)$. The tunnel current (3) then reduces to

$$
J\left(V_{2}, B\right)=\frac{4 \pi N e m^{*}}{h^{2}}|G(0)|^{2}\left|F\left(p_{0}\right)\right|^{2},
$$

so that the magnetocurrent $J\left(V_{2}, B\right)$ directly measures the Fourier distribution $\left|F\left(p_{y}\right)\right|^{2}$ from which $\phi(y)$ may, in principle, be reconstructed. Provided the donor wave function is field-independent and separable so that the transition rates are of the form given in Eq. (2), this result remains true when exact values for the 2DEG energy levels $E_{\mathrm{L}}\left(p_{y}, p_{z}\right)$ are used [9]. Taking $\phi(y)=\exp \left(-y^{2} / \lambda^{2}\right)$ gives a Gaussian field dependence $J\left(V_{2}, B\right) / J\left(V_{2}, 0\right)=\exp \left(-\lambda^{2} p_{0}^{2} / 2 \hbar^{2}\right)$ which is in broad agreement with experiment when $\lambda=100 \AA$ is comparable to the Bohr radius in GaAs and $\Delta s=300 \AA[5]$.

In summary we have calculated the current from a 2 DEG into a zero-dimensional donor state. When the emitter and donor bound state energies are aligned under bias $V_{2}$ the magnetocurrent $J\left(V_{2}, B\right)$ directly measures the Fourier probability spectrum $\left|F\left(p_{y}\right)\right|^{2}$ from which the lateral donor wave function may be deduced.

\section{References}

[1] M.L. Leadbeater, E.S. Alves, L. Eaves, M. Henini, O.H. Hughes, A. Celeste, J.C. Portal, G. Hill, M.A. Pate, J. Phys., Condens. Matter 1, 4865 (1989).

[2] T.M. Fromhold, F.W. Sheard, G.A. Toombs, in: The Physics of Semiconductors, Proc. 20th Internat. Conf. on Physics of Semiconductors, Thessaloniki 1990, Eds. E.M. Anastassakis, I.D. Joannopoulos, Vol: 2, World Scientific, Singapore 1990, p. 1250.

[3] R.K. Hayden, D.K. Maude, L. Eaves, E.C. Valadares, M. Henini, F.W. Sheard, G.A. Toombs, Phys. Rev. Lett. 66, 1749 (1991).

[4] M.W. Dellow, P.H. Beton, C.J.G.M. Langerak, T.J. Foster, P.C. Main, L. Eaves, M. Henini, S.P. Beaumont, C.D.W. Wilkinson, Phys. Rev. Lett. 68, 1754 (1992).

[5] J.W. Sakai, T.M. Fromhold, P.H. Beton, L. Eaves, M. Henini, P.C. Main, F.W. Sheard, in preparation.

[6] T.M. Fromhold, F.W. Sheard, G.A. Toombs, Surf. Sci. 228, 437 (1990).

[7] F.W. Sheard, K.S. Chan, G.A. Toombs, L. Eaves, J.C. Portal, in: 14th Internat. Symp. on GaAs and Related Compounds, Inst. Phys. Conf. Ser. 91, 387 (1988).

[8] R.L. Greene, K.K. Bajaj, Solid State Commun. 45, 825 (1983).

[9] T.M. Fromhold, F.W. Sheard, L. Eaves, to be published. 